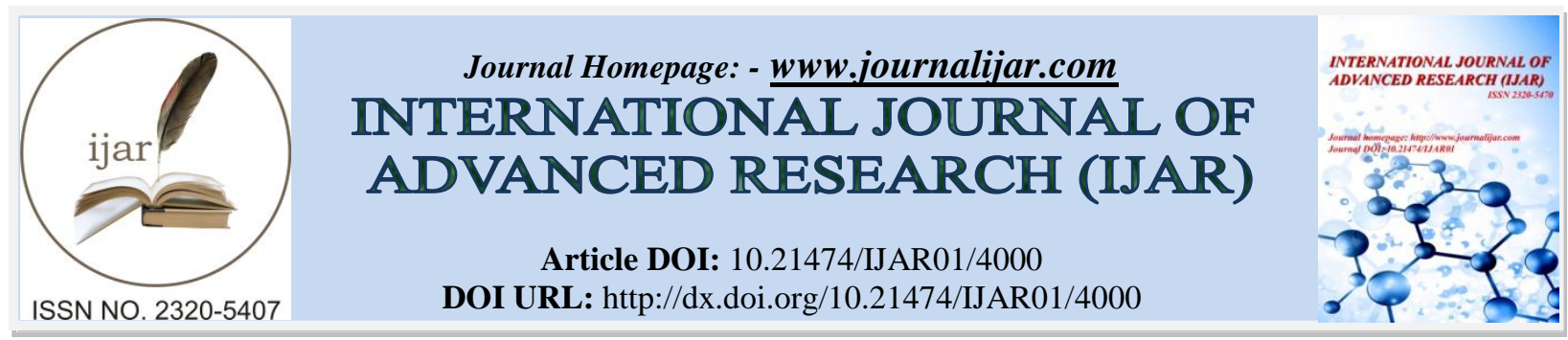

RESEARCH ARTICLE

\title{
KNOWLEDGE, ATTITUDE AND PRACTICE OF HAND SANITATION AMONG THE DENTISTS IN CHENNAI CITY.
}

\section{Harrita. $S^{1 *}$, Dr. Dhanraj ${ }^{2}$ and Dr.Preetham Prasad ${ }^{3}$}

1. Department of prosthodontics, Saveetha University, Chennai-600 077.

2. Head of the department of prosthodontics, Saveetha University, Chennai-600 077.

3. Department of prosthodontics, Saveetha University, Chennai-600 077.

\section{Manuscript Info}

\section{Manuscript History}

Received: 11 February 2017

Final Accepted: 02 March 2017

Published: April 2017

Key words:-

hand sanitation, disease control, hand hygiene guidelines.

\section{Abstract}

Aim: The aim of the study was to evaluate the practice of hand sanitation among the dentists in Chennai city.

Objective: The objective was to evaluate the knowledge and attitude about hand sanitation among the dentist in Chennai city.

Reason: One of the main part of infection control in dentistry is through proper hand sanitation method followed by the dentists. Hand sanitation reduces the risk of transmitting microorganisms, thus reduces the spreading of diseases. This study was done to evaluate the knowledge of dentists about the hand hygiene guidelines that were published by the centre of disease control and protection and their practice of the same.

Materials and method: A cross sectional study was conducted in which 117 dentists, in which 67 were male and 50 were female practicing in Chennai city participated. A self-prepared questionnaire was sent to them and the results were collected and statistics were done. Results:Total of 117 dentists from chennai city participated in the study in which 67 were male and 50 were female. $41.9 \%$ were between the age group $30-50$ years, $38.7 \%$ between the $23-30$ years and $19.4 \%$ were more than 50 years. $64.5 \%$ had a MDS degree and $35.5 \%$ had a BDS degree. $96.8 \%$ dentists agreed that hand sanitation plays an important role in infection control. 58.1\% dentists only knew about the centres of disease control and prevention hand hygiene guidelines. $64.5 \%$ dentists feel that hand sanitisers harm their skin. $61.3 \%$ used plain soap for hand sanitation and only $16.1 \%$ used alcohol based hand sanitisers.

Conclusion: It is important for the dentist to know the infection prevention guidelines, they have to increase their knowledge and also practice proper hand sanitation techniques.Education about proper hand sanitisation through camps, classes and workshops can be conducted. Dentists must encourage fellow dentists, patients and normal public to have proper hand sanitation techniques. 


\section{Introduction:-}

It has been evident for a long time that the health care provider's hand serves as a major source of disease causing pathogens. ${ }^{1,2}$ Morbidity and mortality of patients are many a times linked to the infections from the health care providers. ${ }^{3}$ Hand hygiene is the first step towards providing healthy and infection free treatment. Hand sanitation may sound simple but regular practice of the same among the health care providers is questionable. Association of professionals in infection control has published guidelines for hand washing and hand antisepsis in the year 1988 and 1995. This is similar to the hand washing recommendation given by the centres for disease control and prevention. ${ }^{4,5}$

Proper guideline for hand sanitation gives the health care providers' with careful and rigorous data about hand sanitation and hand antisepsis. It also provides additional information regarding the improved hand hygiene practices and thus decreasing the transmission of harmful microorganisms to the patients and fellow heal care providers. ${ }^{1,6}$ In central Europe alcohol based hand rubs are used since they are proven to have better antimicrobial action. In the US medicated soaps are used. Dentists practicing in India don't follow a proper hand hygiene method regularly.

Thus this study aims to evaluate the knowledge and attitude of dentists practicing in Chennai city towards hand sanitation and also assessing their hand sanitation practice.

\section{Materials and methods:-}

A cross sectional study was conducted by sending questionnaire via electronic mail to dentists practicing in Chennai city. The questionnaire consisted of general demographic details such as age, sex, area of speciality. In order to assess the knowledge and attitude towards hand sanitation questions based on the hand sanitation practice, products they use, attitude towards hand sanitation and their knowledge about the hand sanitation guidelines. The questionnaire were collected and the results were tabulated and statistics were done using SPSS software. 


\section{KNOWLEDGE, ATTITUDE AND PRACTICE OF HAND SANITATION AMONG THE DENTISTS IN CHENNAI} CITY.

\section{KNOWLEDGE:}

1.Do you think there is a connection between hand sanitation and infection control?

- Yes

- No

2.Do you think proper hand sanitation will prevent the spreading of infection?

- Agree

- May be

- Disagree

3.Are you aware about the centres for disease control and prevention hand hygiene guidelines?

- Yes

- No

4.Do you think it is easy to access the centres for disease control and prevention hand hygiene guide?

- Yes

- No

\section{ATTITUDE:}

1.Do you think hand sanitation products harm your skin?

- Agree

- May be

- Disagree

2.Do you think following the hand hygiene guidelines will harm your skin?

- Agree

- May be

- Disagree

3.Do you think your method of hand sanitation is comfortable than the hand hygiene guidelines?

- Agree

- May be

- Disagree

\section{PRACTICE:}

1.Which one of theses you use for your hand sanitation?

- Plain soap

- Antiseptic solution

- Alcohol containing hand sanitiser

2.How long do you was your hands before treating a patient?

- Don't wash

- Wash for less than 5 seconds

- Wash for more than 15 seconds

3. How long do you was your hands after treating a patient?

- Don't wash

- Wash for less than 5 seconds

- Wash for more than 15 seconds

Image 1:- Questionnaire. 


\section{Results:-}

Total of 117 dentists from chennai city participated in the study in which 67 were male and 50 were female. $41.9 \%$ were between the age group 30-50 years, $38.7 \%$ between the $23-30$ years and $19.4 \%$ were more than 50 years. $64.5 \%$ had a MDS degree and $35.5 \%$ had a BDS degree. The overall awareness level is moderate about handsanitation among the dentists.

Image 2: Do you think there is any connection between hand sanitation and infection control?

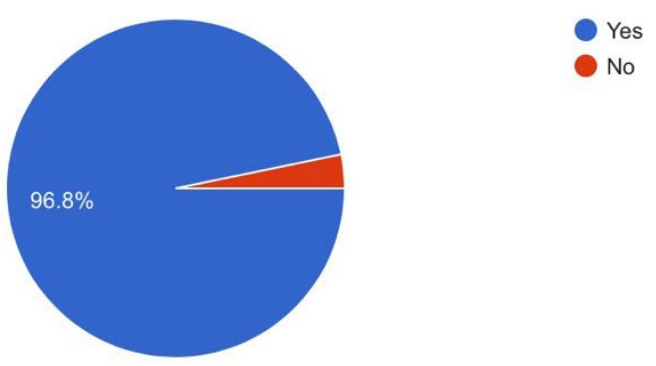

Image 3: Do you think proper hand sanitation will prevent the spreading of infection?

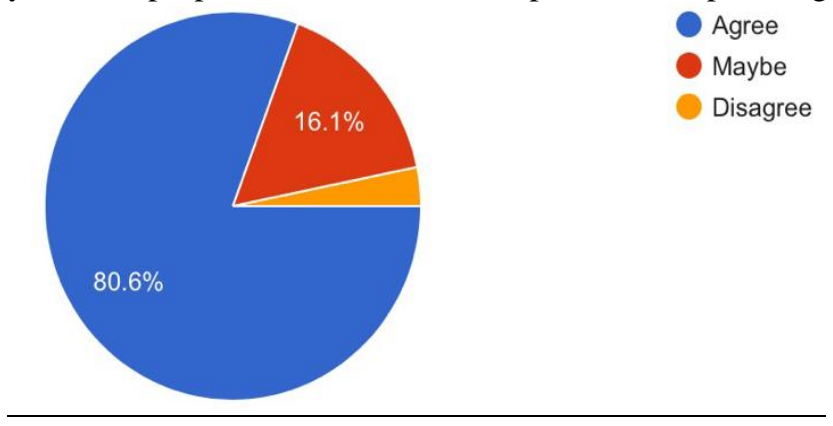

Image 4: Are you aware about the centres of disease control and prevention hand hygiene guidelines?

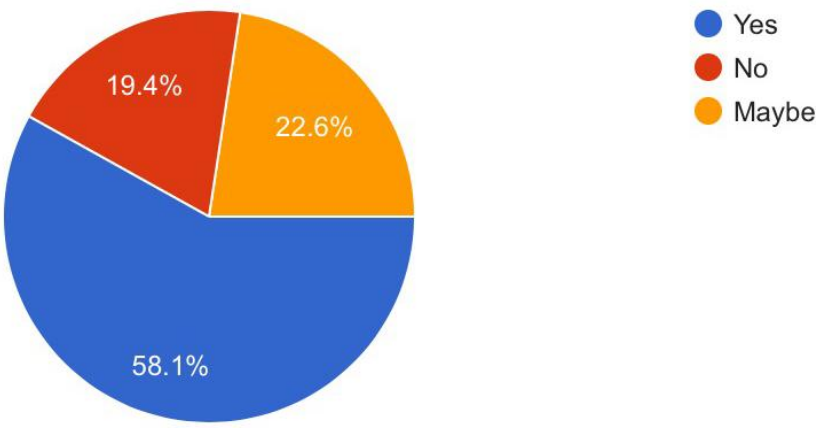


Image 5: Do you think it is easy to access the centres for disease control and prevention hand hygiene guidelines?

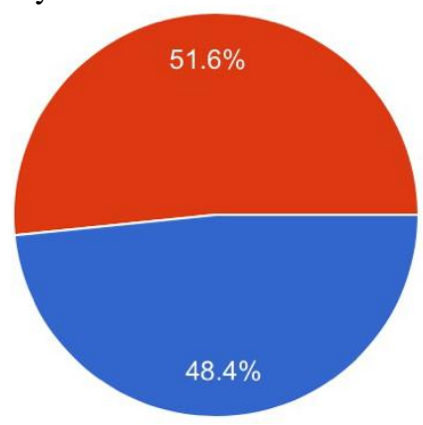

Yes

No

Image 6: Do you think hand sanitation products harm your skin?

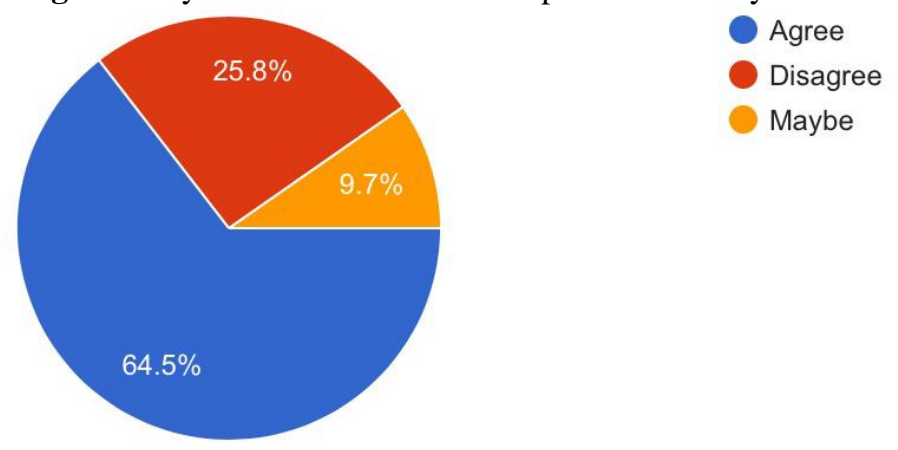

Image 7: Do you think following the hand hygiene guidelines will harm your skin?

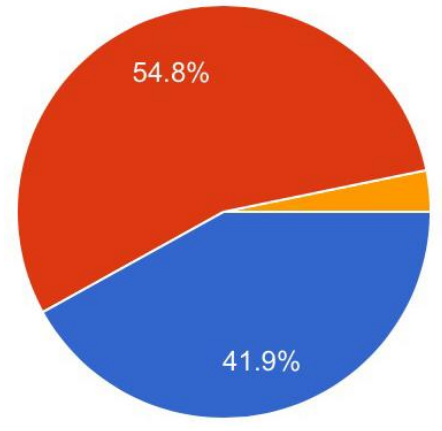

Agree

Maybe

Disagree

Image 8: Do you think your method of hand sanitation is comfortable than the hand hygiene guidelines?

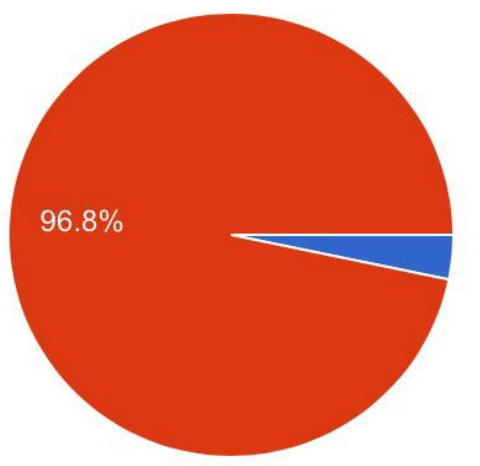

Yes 
Image 9: Have you experienced any allergic reaction by using hand sanitation?

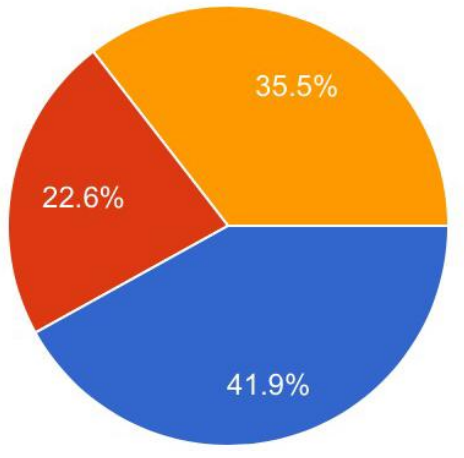

Agree

Disagree

May be

Image 10: Which one of these you use for your hand sanitation?

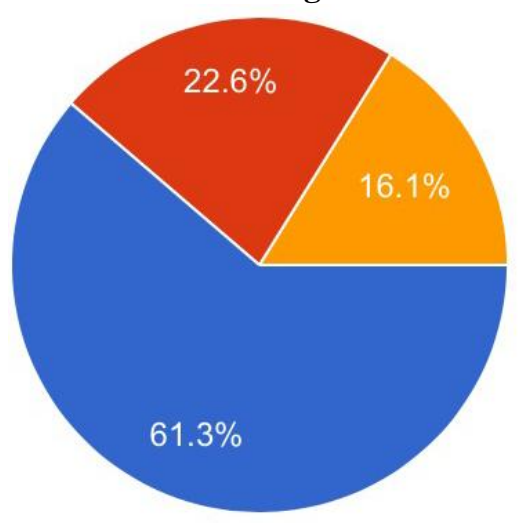

Plain soap

Antiseptic solution

Alcohol containing hand sanitiser

Image 11: How long do you wash your hand before treating a patient?

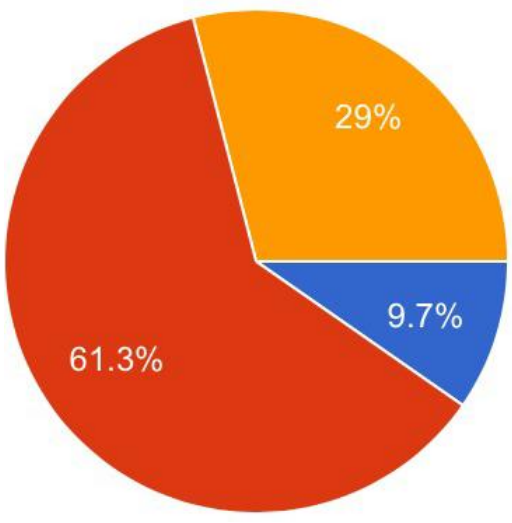

Don't wash

Wash less than 5 seconds

Wash less than 15 seconds 
Image 12: How long do you wash your hands after treating a patient?

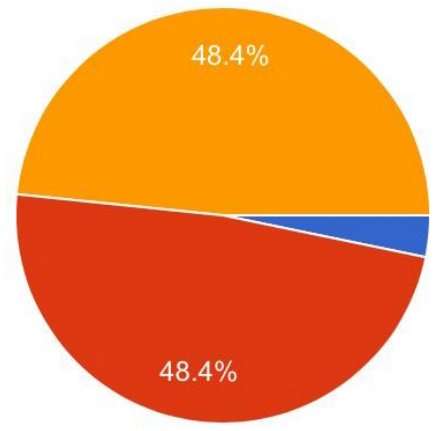

Don't wash

Wash less than 5 seconds

Wash less than 15 seconds

Table 1: LEVEL OF OVERALL AWARENESS

\begin{tabular}{|l|l|l|}
\hline Levels of awareness & Frequency & Percent \\
\hline Inadequate awareness & 15 & 12.9 \\
\hline Moderate adequate awarness & 62 & 53.0 \\
\hline Adequate awareness & 40 & 34.1 \\
\hline Total & 117 & 100.0 \\
\hline
\end{tabular}

\section{Discussion:-}

Dentistry has a need to adhere to scientifically accepted principles of infection control. In the year 2002 the centres for disease control and prevention published hand hygiene in health care settings. ${ }^{1,2}$ The following year the centre for disease control and prevention guidelines for infection control in dental setup, this includedprevious concerns in dentistry like hand sanitation products and Surgical hand sepsis.

Alcohol based hand sanitisersand medicated soaps containing chlorohexidine are considered asideal hand hygiene product.

However alcohols have better antimicrobial activity also unlike the cholorohexidine they are equally Effective against gram positive and gram negative microorganisms, they act by denaturing the protein structure. ${ }^{1}$

About $61.3 \%$ dentists use only plain soap which doesn't remove all the pathogens, a study done by Pottinger $\mathrm{j}$ et al states that Hand washing with plain soap and water for 15 seconds reduces bacterial counts on skin by $0.6-1.1 \log$ $10 .^{7}$

World health organisation recommends alcohol based hand sanitiser because it is fast acting and a broad spectrum of microorganisms can be killed, it can also be used in remote locations where there are minimum amount of resources. ${ }^{8}$

Alcohol based hand sanitisers can be used even when there is only minimum amount of water is available. ${ }^{9,10}$

The main disadvantage of using alcohol based hand sanitiser is it causes dryness of the skin.Only few studies were done to compare the effectiveness of different antiseptics in which most study favoured only cholorohexidine as the better antiseptic. ${ }^{1}$

About $22.6 \%$ of the dentists used antiseptic solution as hand sanitiser and $16.1 \%$ only used alcohol containing hand sanitiser which was similar to the study conducted by Mayers $\mathrm{R}$ et $\mathrm{al}^{2}{ }^{2}$

About $41.9 \%$ dentists were satisfied with their own method of hand sanitation rather than following the CDC's method of sanitation.Pathogens spread quickly when the hand is wet more than when it is dry. ${ }^{11}$ Using soap only removes superficial microflora and fails to completely remove the pathogens adherent to the health care professional's hand. ${ }^{1,11}$

Hand washing must be done before and after treating a patient. It should also be done between while treating the patients if the dentists happen to touch the saliva and other secretion. ${ }^{12}$

Hand sanitation is one of the important part of infection control in dentistry. There are two types of microflora that exists: the transient and the resident.

The transient groups are present in the superficial layer of the skin and are the key cause of infection. 
The resized table group is present in the deep layers and attached to the skin. The choice of hand sanitation must be in such a way that it targets both the group. Wearing gloves reduce the risk of infection by $70-80 \%$ however they have micro leakages through which the transient group of micro flora may escape. ${ }^{1}$

Thus proper hand sanitation is also needed.

In this study most of the dentists used soap and water similar to the study conducted by Sachin Naik et al.

Few dentists also used alcohol based hand sanitation. In General dental procedures there won't be much of infection transmission however in serious surgical procedures proper hand hygiene and protective gears must be worn.

\section{Conclusion:-}

It is important for the dentist to know the infection prevention guidelines, they have to increase their knowledge and also practice proper hand sanitation techniques.Education about proper hand sanitisation through camps, classes and workshops can be conducted. Dentists must encourage fellow dentists, patients and normal public to have proper hand sanitation techniques.

\section{References:-}

1. Sachin Naik, Sanjeev Khanagar, Amit Kumar, Sunil Vadavadagi, Hombesh Mayakonda Neelakantappa, Sujith Ramachandra. Knowledge, attitude, and practice of hand hygiene among dentists practicing in Bangalore city A cross-sectional survey.J Int Soc Prev Community Dent. 2014 Sep-Dec; 4(3): 159-163.

2. Myers R, Larson E, Cheng B, Schwartz A, Da Silva K, Kunzel C. Hand hygiene among general practice dentists: A survey of knowledge, attitudes and practices. J Am Dent Assoc. 2008;139:948-57.

3. Mani A, Shubangi AM, Saini R. Hand hygiene among health care workers. Indian J Dent Res. 2010;21:115-8.

4. Larson E. Status of practice guidelines in the United States: CDC guidelines as an example. Prev Med. 2003;36:519-24.

5. Boyce JM, Pittet D. Guideline for Hand Hygiene in Health-Care Settings Recommendations of the Healthcare Infection Control Practices Advisory Committee and the HICPAC/SHEA/APIC/IDSA Hand Hygiene Task Force Society for Healthcare Epidemiology of America/Association for Professionals in Infection Control/Infectious Diseases Society of America. MMWR Recomm Rep. 2002;5:1-45. quiz CE1-4.

6. Houben E, De Paepe K, Rogiers V. Skin condition associated with intensive use of alcoholic gels for hand disinfection: A combination of biophysical and sensorial data. Contact Dermatitis. 2006;54:261-7.

7. Pottinger J, Burns S, Manske C. Bacterial carriage by artificial versus natural nails. Am J Infect Control 1989;17:340-4.

8. WHO Guidelines on Hand Hygiene in Health Care (Advanced Draft): A Summary. World Health Organization; 2005.

9. Simmons B, Trusler M, Roccaforte J, Smith P, Scott R. Infection control for home health. Infect Control Hosp Epidemiol 1990;11:362-70.

10. Steere AC, Mallison GF. Hand washing practices for the prevention of nosocomial infections. Ann Intern Med 1975;83:683-90.

11. Patrick DR, Findon G, Miller TE. Residual moisture determines the level of touch-contact-associated bacterial transfer following hand washing. Epidemiol Infect 1997;119:319-25.

12. Ehrenkranz NJ, Alfonso BC. Failure of bland soap handwash to prevent hand transfer of patient bacteria to urethral catheters. Infect Control Hosp Epidemiol. 1991;12:654-62. 